\title{
Success of Immediate Implants in Anterior Esthetic Zone: One Year Prospective Study
}

\author{
${ }^{1}$ Saurabh Sathe, ${ }^{2}$ Khalid Gufran, ${ }^{3}$ Mariam OB Hamza, ${ }^{4}$ Inderjit M Gowdar, ${ }^{5}$ Mohammed AA Elqomsan, ${ }^{6}$ Mubashir B Mirza
}

\section{ABSTRACT}

Aim: The present study was aimed to assess the success of immediate implants in the anterior esthetic zone.

Materials and methods: Fifteen patients with 15 teeth to be replaced were selected for the study. After extraction, the socket was thoroughly cleaned and curetted with betadine and saline. Using physiodispenser and drills socket was prepared for implant insertion and implants were placed with initial stability with hand motion followed by complete insertion with hand ratchet. The socket was closed with 3-0 silk and medications were given with instructions to care for wound and recall after 1 week. The variables were measured postoperatively according to clinical parameters, i.e., mobility, exudate, pain, patient satisfaction score at a regular interval of 1, 3, 6 and 12th month. A $p$ value of $<0.05$ was considered as statistically significant. Qualitative data were analyzed using the Fischer exact test.

Results: The mean age was found to be 23.33 years (range 14-38 years). There were $13(86.6 \%)$ males and $2(13.4 \%)$ females. Central incisors were replaced in $12(80 \%)$ patients, lateral incisors were replaced in $2(13.33 \%)$ patient and $1^{\text {st }}$ premolar was replaced in $1(6.66 \%)$ patient. No pain, exudates was experienced by any of the patients in all 4 follow up scheduled. Only $2(13.33 \%)$ implant which was experienced mobility of grade 1 at 12 months follow-up. Three (20\%) patients had satisfaction scores of $8,6(40 \%)$ had a satisfaction score of 9 and $6(40 \%)$ had a score of 10 . Thus, the majority of patients were very happy with the implants placed.

Conclusion: In conclusion, the present study has shown that immediate placement of implants in the anterior esthetic zone has a predictable success rate with good patient acceptance.

Clinical significance: Clinically, placing an implant at the time of extraction has more advantages like healing time reduction, helps maintain alveolar architecture, surgical interventions will be decreased. So it helps enhance the success rate.

${ }^{1}$ Department of Oral and Maxillofacial Surgery, Bhabha College of Dental Sciences, Bhopal, Madhya Pradesh, India

${ }^{2,4}$ Department of Preventive Dental Sciences, College of Dentistry, Prince Sattam Bin Abdul Aziz University, Alkharj, Riyadh, Kingdom of Saudi Arabia

${ }^{3}$ Department of Dentistry, Alkharj Armed Forces Hospital, Alkharj, Riyadh, Kingdom of Saudi Arabia

${ }^{5,6}$ Department of Conservative Dental Science, College of Dentistry, Prince Sattam Bin Abdul Aziz University, Alkharj, Riyadh, Kingdom of Saudi Arabia.

Corresponding Author: Saurabh Sathe, Department of Oral and Maxillofacial Surgery, Bhabha College of Dental Sciences, Bhopal, Madhya Pradesh, India, Phone: +919993952603, e-mail: drsaurabhsathe@gmail.com
Keywords: Esthetic zone, Immediate implants, Missing tooth, Prospective study.

How to cite this article: Sathe S, Gufran K, Hamza MOB, Gowdar IM, Elqomsan MAA, Mirza MB. Success of Immediate Implants in Anterior Esthetic Zone: One Year Prospective Study. World J Dent 2018;9(6):451-456.

Source of support: Nil

Conflict of interest: None

\section{INTRODUCTION}

The problem of the missing tooth has troubled mankind ever since times immemorial, historic solutions included replacement by cadaveric teeth, animal teeth, artificial teeth made of vulcanite and metal which could be fixed to adjacent teeth or removed by the user. With advancement in material sciences and improvement of our understanding of occlusion and gnathostomatic system, better modalities of tooth replacement came into existence with three primary goals of comfort, function, and esthetics. The concept of a dental implant for replacement of missing tooth came into existence after professor Branemark gave the concept of osseointegration in $1952 .^{1}$

By traditional protocols, the dental implant once placed in the bone and submerged under mucosa is left for a healing time of 3-6 months that ensures proper osseointegration between bone and implant. During this stage the patient is advised to wear an interim prosthesis and later in the 2nd stage, abutment was attached to the implant and after proper soft tissue healing the process of final prosthesis generation was start. The impending loss of a single tooth in the esthetic zone in a patient with an otherwise healthy periodontium can be a distressing experience. $^{2}$

In recent years, immediate implant placement after tooth extraction has become a common clinical therapeutic approach, an alternative to a staged surgical protocol. The reduction in the number of surgeries needed and the advantage of a shorter time to rehabilitate function and aesthetic has provided an impetus to studies on this surgical approach. Clinical studies have demonstrated that the survival rates for implants placed immediately, early, delayed or late seem to be similar in short-term follow-ups and range between $93 \%$ and $100 \% .^{3}$ 
Douglas $^{4}$ reported that placing immediate implants ought to have three- and four-walled sockets, with less resorption of periodontal bone, sufficient bone available to stabilize implant, and to reduce circumferential defects. When these were met, placement of immediate implant could be a viable and safe procedure. Schwartz-Arad and Chaushu ${ }^{5}$ have placed 95 implants immediately and studied for five years, with a success rate of $95 \%$. Hence this 1-year prospective study was conducted with an aim to assess the success of immediate implants in the anterior esthetic zone.

\section{MATERIALS AND METHODS}

\section{Source of Data}

The patients for this prospective study were selected from the outpatient department of oral and maxillofacial surgery, Government College of Dentistry, Indore. The study was conducted on subjects from the local population who required placement of single or multiple missing teeth in the anterior esthetic zone after removal of the hopeless tooth. Total 15 implants were placed. Before commencement of implant surgery a detailed history of patients were taken, the patients were explained about the potential risks and benefits associated and informed consent was obtained.

\section{Patient Selection}

Fifteen patients with 15 teeth to be replaced were selected for the study (patients required tooth extraction in the anterior esthetic zone that was from right premolar to left premolar).

Age 14 or more with permanent dentition, the presence of at least one poor prognosis anterior tooth (incisor, canine, premolar by-trauma, root resorption, caries, endodontic failure). Presence of adequate gingival architecture with surrounding dentition. Presence of at least

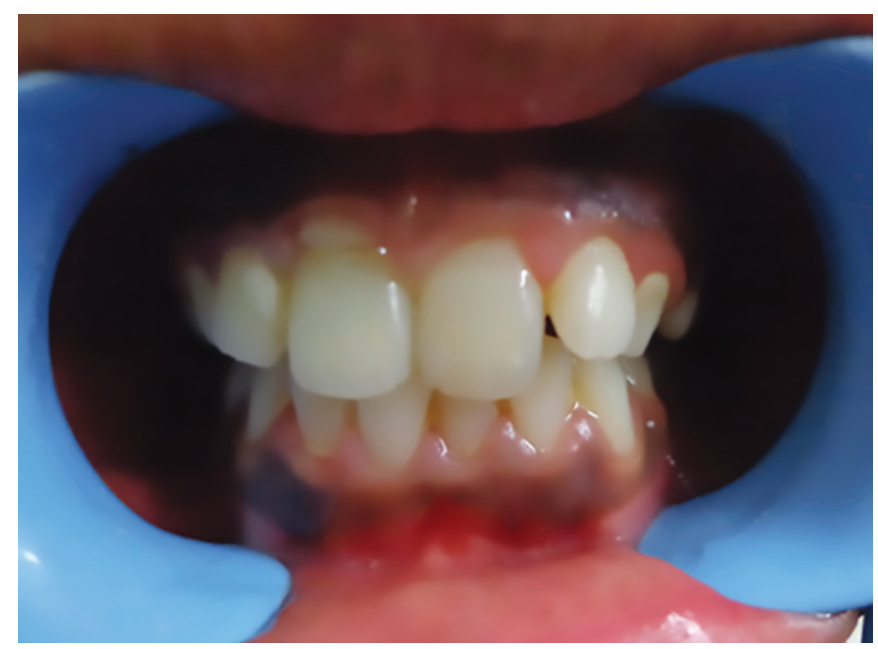

Fig. 1: Before extraction of the tooth
$4 \mathrm{~mm}$ of bone beyond root apex and the adjacent tooth, patients with no systemic or chronic disease, good oral hygiene were included in the study. Presence of uncontrolled diabetes, coagulation disorders, acute infection around the tooth, heavy smoking, drug abuse history, poor oral hygiene or any systemic diseases participants were excluded.

\section{Surgical Procedure}

Patient selection was done according to the inclusion and exclusion criteria of the study. The surgical procedure was done on a dental chair at the Department of Implantology. Government College of Dentistry (GDC) Indore. All patients were firstly draped and painted with betadine. Patients were given $2 \%$ lignocaine with adrenaline (lignox) at nasopalatine foramen and infraorbital regions. After achieving anesthesia atraumatic extraction was done with the help of luxators and other instruments (Figs 1 and 2). Care was taken not to damage the socket wall while extraction and avoiding excessive force with conservation of gingival margins.

After extraction, the socket was thoroughly cleaned and curetted with betadine and saline. Using physiodispenser and drills socket was prepared for implant insertion taking care of irrigation with saline to avoid heating up of socket while drilling. Implants were then placed with initial stability with hand motion followed by complete insertion with hand ratchet (Fig. 3). The socket was closed with 3-0 silk and medications were given with instructions to care for wound and recall after 1 week.

After cutting sutures 1 week later, a removable prosthesis was planned for the patient until stage 2. At the end of 3 months wound was opened and the gingival former was placed for 15 days after which impression was taken and sent to lab and crown was fixed within a week (Fig. 4). Regular follow-up and IOPA were taken to check results and patient satisfaction.

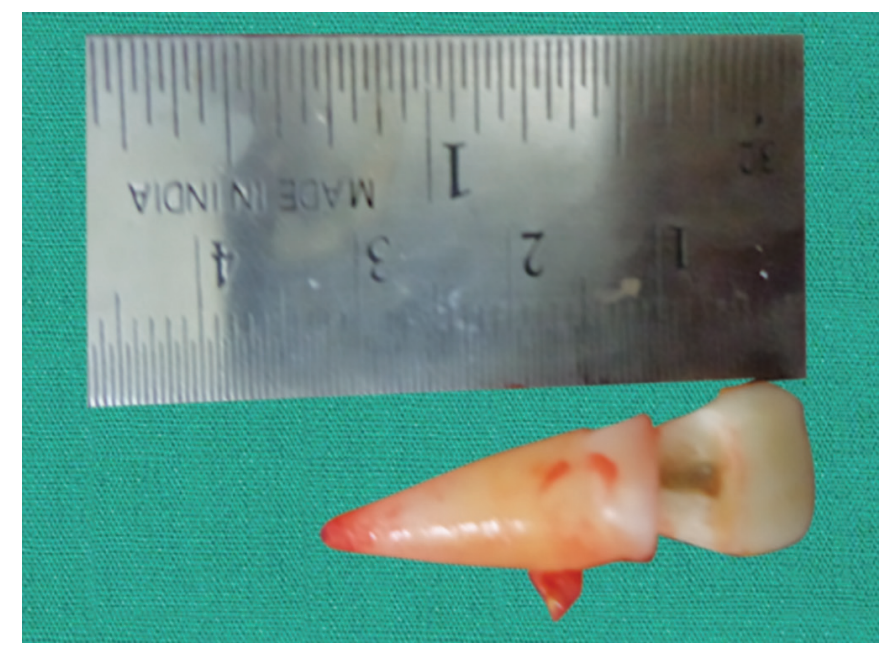

Fig. 2: Measurement of the tooth length 


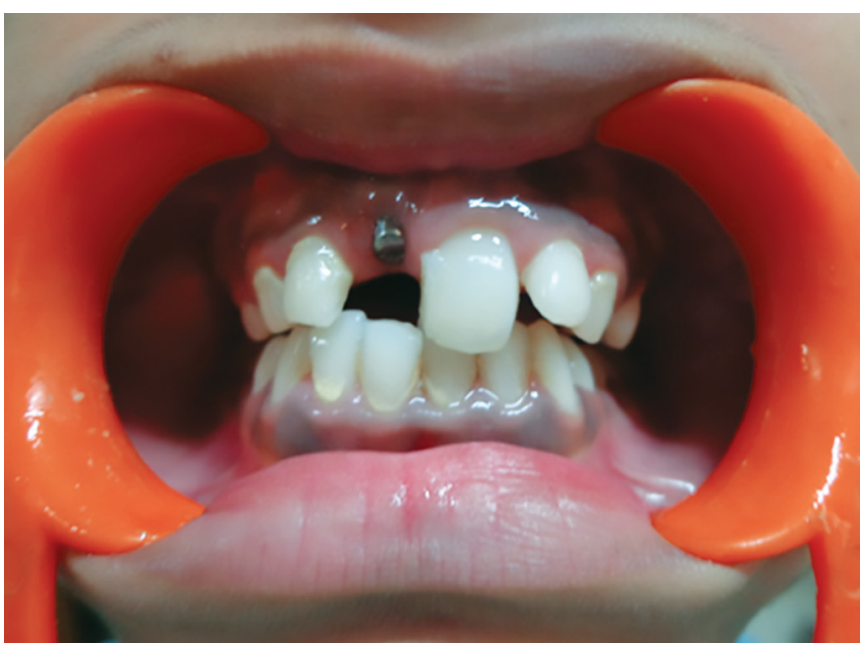

Fig. 3: Placement of cover screw

\section{Data Collection}

Evaluation to be made after implant placed at an interval of 1st, 3rd, 6th and 12th months.

Implant success or failure-defined under 4 categories as per ICOI (international congress of oral implantologists) PISA consensus.

\section{- Success (Optimum health)}

${ }^{*}$ No pain or tenderness upon the function

*Zero mobility

${ }^{*}$ No exudates history

\section{- Satisfactory Level}

${ }^{*}$ No pain on function

*Zero mobility

${ }^{*}$ Exudates history

\section{- Compromised Level}

${ }^{*}$ May have sensitivity on function

*No mobility

*Exudates history

\section{- Failure (Clinical or absolute failure)}

*Pain on function

${ }^{*}$ Mobility

*Uncontrolled exudates

*Out of the mouth

The variables were measured postoperatively according to clinical parameters to assess the objectives of the study

\section{Mobility}

It was measured in a way similar to measure tooth mobility, using two rigid instruments with a force of approx $500 \mathrm{~g}$ were applied in labiolingual direction. The amount of mobility was measured in a score of $0-4$.

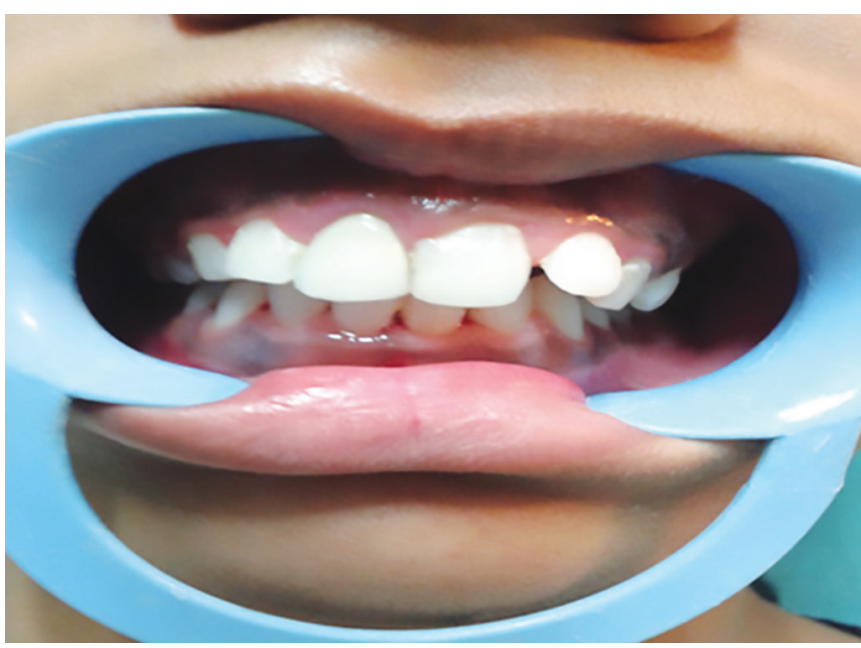

Fig. 4: Final placement of implant crown

\section{Implant Mobility Scale}

0 The absence of any clinical mobility with $500 \mathrm{~g}$ in any direction

2 Horizontal slight detectable mobility

3 Horizontal visible moderate mobility

4 Horizontal severe mobility $>0.5 \mathrm{~mm}$

5 Visible moderate to severe horizontal mobility

\section{Exudate}

The presence of any visible exudates flow or pus discharge around the prosthesis on examination or by the patient during the function.

\section{Pain}

To assess the implanted pain, percussion method was used up to $500 \mathrm{~g}(1.2 \mathrm{psi})$.

\section{Patient Satisfaction Score}

After 12 months follow-up patient was asked

- On scale $0-10$, where 0 is unsatisfied and 10 are totally satisfied, what scores you will give for the satisfaction with the esthetic outcome of your treatment.

- Did you recognize the changes in gum level around the implant?

- Patients were recalled after delivery of prosthesis at a regular interval of 1, 3, 6 and 12th month to evaluate the parameters associated with implant stability and success. Each patient's parameters were measured postoperatively.

\section{RESULTS}

Table 1 depicts the distribution of patients according to gender. There were $13(86.6 \%)$ males and $2(13.4 \%)$ females. There was a male preponderance in the present study patients. The mean age was found to be 23.33 years (range 14-38 years). 
Table 1: Distribution of patients according to gender

\begin{tabular}{lll}
\hline Gender & Number & Percentage \\
\hline Male & 13 & $86.6 \%$ \\
Female & 2 & $13.4 \%$ \\
\hline Total & 15 & $100 \%$ \\
\hline
\end{tabular}

Table 3: Distribution of implants according to pain assessment at different follow-up schedules

\begin{tabular}{lllll}
\hline & \multicolumn{3}{c}{ Pain Assessment } \\
\cline { 2 - 5 } Follow-up Visits & No & $\%$ & No & $\%$ \\
\cline { 2 - 5 } 1 Month & 0 & 0.00 & 15 & 100.00 \\
3 Month & 0 & 0.00 & 15 & 100.00 \\
6 Month & 0 & 0.00 & 15 & 100.00 \\
12 Month & 0 & 0.00 & 15 & 100.00 \\
\hline Fischer exact test $\mathrm{x}^{2}=5.810, \mathrm{p}=0.621$ &
\end{tabular}

Table 2 reveals the distribution according to tooth replaced. Central incisors were replaced in $12(80 \%)$ of the patients, lateral incisors were replaced in $2(13.33 \%)$ of the patient and 1st premolar was replaced in $1(6.66 \%)$ of the patient.

The pain assessment at different follow-up visits was shown in Table 3. No pain was experienced by any of the patients in all 4 follow-up studied scheduled at 1, 3, 6 , and 12 month and there was no significant difference between the intervals.

No exudates were experienced by any of the patients in all 4 follow-up studied scheduled at 1, 3, 6, and 12 months and a significant difference was not found between the intervals (Table 4).

The mobility assessment at different follow-up visits shown in Table 5. Except for 2 (13.33\%) implant which experienced mobility of grade 1 at 12 months follow-up, no mobility was experienced by any of the patients in all 4 follow-up studied scheduled at 1, 3, 6 and 12 months and there was no significant difference between the intervals.

Three $(20 \%)$ patients had satisfaction scores of 8,6 $(40 \%)$ had a satisfaction score of 9 and $6(40 \%)$ had a score of 10 . Thus, the majority of patients were very happy with the implants placed (Graph 1).

\section{Statistical Analysis}

In the current study, a descriptive analysis was done. Results on categorical measurements are presented in

Table 5: Distribution of implants according to "Mobility" at different follow-up schedules

\begin{tabular}{lllll}
\hline & \multicolumn{4}{c}{ Mobility assessment } \\
\cline { 2 - 5 } Follow-up & \multicolumn{3}{c}{ Present } & \multicolumn{3}{c}{ Absent } \\
\cline { 2 - 5 } Visits & No & $\%$ & No & $\%$ \\
\hline 1 Month & 0 & 0.00 & 15 & 100.00 \\
3 Month & 0 & 0.00 & 15 & 100.00 \\
6 Month & 0 & 0.00 & 15 & 100.00 \\
12 Month & 2 & 13.33 & 13 & 86.67 \\
\hline
\end{tabular}

Fischer exact test $X^{2}=6.895, p=0.512$
Table 2: Distribution of implants according to tooth replaced

\begin{tabular}{lll}
\hline Tooth replaced & Number & Percentage \\
\hline Central incisor & 12 & 80 \\
Lateral incisor & 2 & 13.33 \\
First premolar & 1 & 6.67 \\
\hline Total & 15 & 100 \\
\hline
\end{tabular}

Table 4: Distribution of implants according to "Exudates" at different follow-up schedules

\begin{tabular}{lllll}
\hline & \multicolumn{4}{c}{ Exudates Assessment } \\
\cline { 2 - 5 } Follow-up Visits & No & $\%$ & No & $\%$ \\
\cline { 2 - 5 } 1 Month & 0 & 0.00 & 15 & 100.00 \\
3 Month & 0 & 0.00 & 15 & 100.00 \\
6 Month & 0 & 0.00 & 15 & 100.00 \\
12 Month & 0 & 0.00 & 15 & 100.00 \\
\hline Fischer exact test $\mathrm{x}^{2}=6.321, \mathrm{p}=0.154$ & &
\end{tabular}

number (\%). The significance is assessed at $5 \%$ level of significance with a 95\% confidence interval. Qualitative data were analyzed using the Fischer exact test.

\section{DISCUSSION}

The history of dental implants goes way back in time. From ancient times human civilizations have been trying to replace missing tooth with more other substitutes like ivory, stone, shells, other human and even animal teeth With time and development in dentistry the concept of RPD and FPD was introduced and it served as a suitable replacement of lost tooth for a long time, but it was never a match for loss of functional capacity, stability, masticatory efficiency that of a natural tooth plus the alveolar bone loss that take place progressively was also a concern. ${ }^{6}$

- Branemark et al. ${ }^{1}$ completely change the concept of dental implants with the concept of osseointegration which he described as "a stable contact zone between the bone and implant surface without interposing soft tissue under the light microscope."

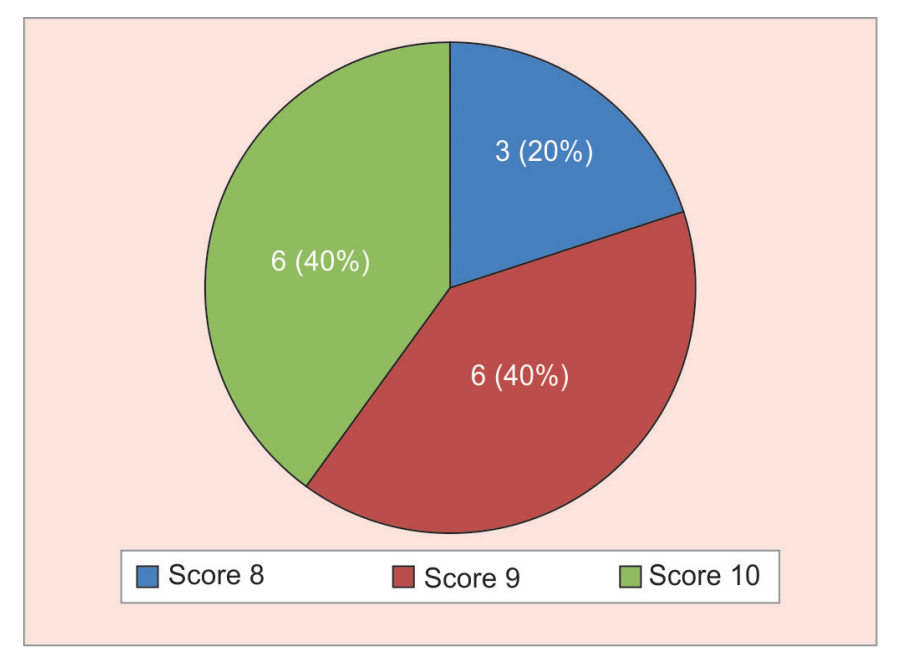

Graph 1: Distribution of implants according to patient satisfaction scores 
- Adell et al. ${ }^{7}$ conducted a study states that extraction site needs 8-12 months for proper healing and socket ossification, after that the implant was placed and left for 4-6 months to get osseointegrated with alveolar bone. Then the prosthetic phase was started with exposure of implant surface and prosthesis was made. The success rate of implants was as high as $90 \%$ and it was considered the best option in prosthetic replacement as compared to natural teeth.

- Schropp et al. ${ }^{8}$ showed in his study that significant ridge resorption occurs 12 months after extraction with $2 / 3$ rd of changes occurring in the first 3 months.

- In the current study, the immediate implant success rate was good with patient satisfaction. Which was similar to the study done by Rosenquist et al. ${ }^{9}$ who did immediate placement of 109 implants with a success rate of $92 \%$. The immediate implants showed that placing an implant in a freshly extracted socket prevents alveolar bone resorption especially on the buccal side which has a thin bone. The patient satisfaction was also improved as this procedure offered few surgeries as compared to traditional protocol and decreased edentulous span.

- Covani et al ${ }^{10}$ reported that a total of 35 implants; 20 were immediately placed after tooth extraction and 15 implants placement was done in-between 4 and 6 weeks after the tooth extraction. Primary flap closure was done and implants were submerged in it. At reopening in both groups showed similar kind of healing and bone fill at the coronal portion of the implants.

- Ganeles and Wismeijer ${ }^{11}$ assessed the outcome with different loading periods and conditions (implants stability, multiple teeth vs. single tooth and bone quality). And finally, it showed a success rate of $98.2 \%$. Cornelini et al. ${ }^{12}$ conducted a study with 22 implants and a 12-month follow-up period reveals $0.5 \mathrm{~mm}$ of bone resorption at 12 months.

Presence of pain is a criterion included in the current study for evaluating the success of immediate implants. In a success and failure areas, it was assumed that the implant should not injure the major nerves of the jaws. ${ }^{7}$ In this study, no pain was experienced by any of the patients in all the four follow-up visits that were scheduled at 1 , 3, 6, and 12 months.

Presence of mobility was another factor evaluated in the current study. Except for 2 (13.33\%) implant which experienced mobility of grade 1 at 12 months follow-up, no mobility was experienced by any of the implants at all the four follow-up visits that were scheduled at 1, 3, 6 , and 12 months. This because the patient has applied forces on the system in the early healing period and this generates micromotions. The presence of micromovements at the interface between bone and implant can affect bone healing and osseointegration, leading to a slight mobilization of the implant.

- Sekine et al. ${ }^{13}$ states that the movement of the healthy implant should be less than 75 micrometers and its considered as zero clinical mobility. Another study conducted by Winkler et al. ${ }^{14}$ states that the presence of connective tissue between the implant and bone has considered as a clinical failure for an endosteal root-form implant.

In the present study, no exudates were experienced by any of the patients in all the four follow-up visits that were scheduled at $1,3,6$, and 12 months.

- Mombelli A et al. ${ }^{15}$ explains exudates remain for more than one to two weeks requires surgical invasion of the peri-implant area to remove causative organism.

Patient satisfaction score was evaluated in the present study at end of the 12th month. The maximum score was 10 and the patient was asked to rate his/her experience of the implant and prosthesis regarding function, appearance and personal satisfaction. Three (20\%) patients had a satisfaction score of $8,6(40.0 \%)$ patients had a satisfaction score of 9 and $6(40 \%)$ had a satisfaction score of 10 . Thus, shows that majority of the patients were very happy with the implants placed.

To get a better rate of success most important is case selection, patients with smoking and other tobacco habits are always a poor contender. Same can be said for patients with a systemic disease like diabetes and any diseases affecting bone remodeling and structure. Doing atraumatic extractions and using bone grafts and membrane can increase chances of success in cases with more loss of buccal cortical bone. Using better quality implants with more features which can increase the osseointegration and can make immediate loading of implants which can provide patients a functioning occlusion soon after the loss of teeth.

Limitations include patients with severe trauma with dentoalveolar fracture placing implants is very difficult with low chances of success, Economic hurdles, Patients with smoking habits and parafunctional habits are also poor contenders for the procedure.

Even though the study shows encouraging results regarding clinical outcomes and patient satisfaction level, still results are derived from a small sample group of 15 implants with a brief follow-up, further studies regarding other aspects to measure success of immediate implants are needed with larger sample size to give another inch of edge to concept of immediate implants to prove it as a better and suitable replacement of traditional delayed implant protocol. 


\section{CONCLUSION}

In conclusion, the present study has shown that immediate placement of implants in the anterior esthetic zone has a predictable success rate with good patient acceptance. Immediate implants have emerged as a valuable treatment option for missing teeth successfully overcoming the disadvantages associated with the traditional delayed implant protocols.

\section{REFERENCES}

1. Branemark P I. Osseointegration and its experimental background. J Prosthet Dent 1983;50:399-410.

2. Kan JY, Rungcharassaeng K, Lozada J. Immediate Placement and Provisionalization of Maxillary Anterior Single Implants: 1-Year Prospective Study. Int J Oral Maxillofac Implants. 2003;18(1):31-39.

3. Simsek B, Simsek S. Evaluation of success rates of immediate and delayed implants after tooth extraction. Chin Med J. 2003; 116(8):1216-1219.

4. Douglass GL, Merin RL. The immediate dental implant. J Calif Dent Assoc 2002;30:362.

5. Schwartz-Arad D, Chaushu G. The ways and wherefores of immediate placement of implants into fresh extraction sites: A literature review. J Periodontol. 1997;68(10):915-923.

6. Ring ME. A thousand years of dental implants: a definitive history--part 1. Compendium of continuing education in dentistry (Jamesburg, NJ: 1995). 1995 Oct;16(10):1060-1062.

7. Adell R, Eriksson B, Lekholm U, Branemark PI, Jemt T. A long-term follow-up study of osseointegrated implants in the treatment of totally edentulous jaws. Int J Oral Maxillofac Implants. 1990 Winter;5(4):347-359.

8. Schropp L, Wenzel A, Kostopoulos L, Karring T. Bone healing and soft tissue contour changes following single-tooth extraction: a clinical and radiographic 12 -month prospective study. International Journal of Periodontics and Restorative Dentistry. 2003 Aug 1;23(4):313-323.

9. Rosenquist B, Grenthe B. Immediate placement of implants into extraction sockets: implant survival. Int J Oral Maxillofac Implants. 1996 Mar-Apr;11(2):205-209.

10. Covani U, Bortolaia C, Barone A, Sbordone L. Bucco-lingual crestal bone changes after immediate and delayed placement. J Periodontol. 2004;75:1605-1612.

11. Ganeles J, Wismeijer D. Early and immediately restored and loaded dental implants for single-tooth and partial-arch applications. Int J Oral Maxillofac Implants. 2004;19 Suppl: 92-102.

12. Cornelini R, Cangini F, Covani U. and Wilson T G Jr. Immediate restoration of implants placed into fresh extraction sockets for single-tooth replacement: a prospective clinical study Int J Periodontics Restorative Dent. 2005 Oct;25(5): 439-447.

13. Sekine H, Komiyama Y, Hotta H, Yoshida K. Mobility characteristics and tactile sensitivity of osseointegrated fixturesupporting systems. Tissue integration in oral maxillofacial reconstruction. In "Van Steenberghe", Elservier, Amsterdam. 1986:326-332.

14. Winkler S, Morris HF, Spray JR. Stability of implants and natural teeth as determined by the Perio test over 60 months of function. J Oral Implantol. 2001;27:198-203.

15. Mombelli A, Lang NP. The diagnosis and treatment of periimplantitis. Periodontol 2000. 1998;17:63-76. 